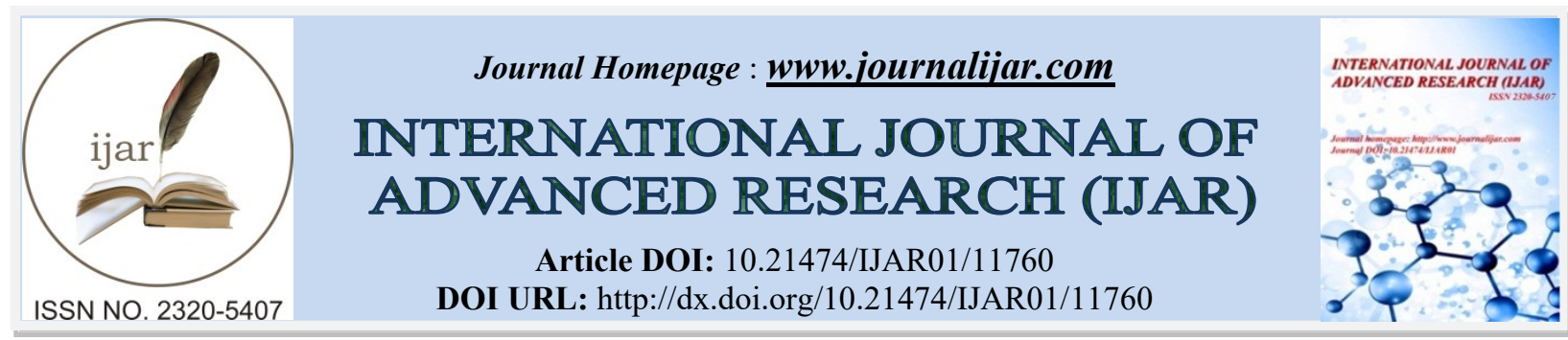

RESEARCH ARTICLE

\title{
AYRES SENSORY INTEGRATION FOR THE CHILDREN WITH ATTENTION DEFICIT AND HYPERACTIVITY DISORDER (ADHD):A MIXED METHOD STUDY
}

\author{
Nandgaonkar Hemant $\mathbf{P}^{1}$ and Zarine Ferzandi ${ }^{2}$ \\ 1. Assistant Professor, Seth GS Medical College, KEM Hospital, Mumbai. \\ 2. Associate Professor (Retired), Seth GS Medical College, KEM Hospital, Mumbai.
}

\section{Manuscript Info}

..........................

Manuscript History

Received: 20 July 2020

Final Accepted: 24 August 2020

Published: September 2020

Key words:-

ADHD Participation Profile, Children, Goal Attainment Scale, Ayres Sensory Integration

\section{Abstract}

For intervention of children with a diagnosis of Attention deficit hyperactivity disorder (ADHD), there are many modalities. Sensory Processing issues are present in children with ADHD. There is a wide use of Ayres Sensory Integration (ASI $\left.{ }^{\circledR}\right)$ to treat children with ADHD. But effectiveness of ASI ${ }^{\circledR}$ in children with ADHD is unknown. The purpose of study was to examine the effects of ASI ${ }^{\circledR}$ Interventions on Participation of the child in addition to the parent's perspective about the outcome of therapy in children with a diagnosis of ADHD.

Method: Study was divided as

1. Development of the questionnaire for the evaluation of "Participation of the child with ADHD".

2. Randomized controlled Pilot trial, double blind study.

3. Family perspective about the effect of intervention on children with ADHD using qualitative interview method.

Eligible candidates were children having average intelligence with a diagnosis of ADHD, between age of 5 years and 12 years, sensory processing disorder. Children included in the study received occupational therapy with either protocol.

Results:

1. Self-care, academics, interpersonal interactions and communication are important primacies for the parents of children with a diagnosis of ADHD.

2. Priorities of the parents of children with a diagnosis of ADHD change according to the culture they belong.

3. ADHD Participation Profile (APP) is a valid \& reliable questionnaire to monitor progress of children with a diagnosis of ADHD.

The responsiveness of the ADHD Participation Profile questionnaire has been proven to be good. Herein, the ADHD Participation Profile can be used reliably to document the treatment progress of patients with ADHD for research and clinical purposes. The children in ASI® Group, made gains that were significantly greater than the children in the other group on Goal Attainment Scale and ADHD Participation 
Profile. Large Effect sizes were found between and within the on both the outcome measures $(1.87 \& 0.939)$.

Conclusion: ADHD does not have only represents sensory processing issues. It's is complex disorder which embodies issues other than Sensory Processing and will be receptive to the other modes of intervention. If existing, the sensory processing issues needs to be addressed specifically, as it will influence the global performance. Occupational Therapy has brought a notable change in kids and parent's life. Most of the caretakers were ready for new boundaries in their daily family occupations. Though some goals are met after the intervention, the list of apprehensions is unending. Many parents less worried after Occupational Therapy intervention, whereas the few were still doubtful about future of their kids. We would have overlooked this ironic data, if we should have given a verdict about the effect of the intervention though quantitative measure. Mixing the methods was a suitable solution.

Copy Right, IJAR, 2020,. All rights reserved.

\section{Introduction:-}

Attention deficit hyperactivity disorder (ADHD) is a highly prevalent childhood disorder. ADHD impacts the participation of children in various life circumstances. It may be school, playground, home or some other community environment. Very frequently, the usual signs of impulsivity, inattention and hyperactivity prohibit the child from engaging in many scenarios. There are many ways of treating these children. These children are commonly referred to Occupational Therapy. Occupational Therapist use Ayres Sensory Integration ${ }^{\circledR}$ (ASI) Therapy very widely for intervention. But it is still considered an unproven intervention. In this context, it is very interesting to research the influence of ASI on the involvement of children in different life situations.

In many studies, researcher take into account the existence of sensory modulation disorders (SMDs). Certain types of SPD, Sensory Based Modulation Disorder (SBMD) also contribute to the attention and organization of behavioral problems reported in children with ADHD. It is therefore necessary to assess SPD (sensory processing disorder) in general rather than to consider SPD (sensory modulation disorder) only. In this sense, all children diagnosed with ADHD should have a thorough assessment of sensory processing. After the evaluation and confirmation of the sensory processing disorder, Ayres Sensory Integration ${ }^{\circledR}$ Therapy intervention should begin.

Also, most of the studies evaluate outcome on standardized scales using quantitative methods. But it is equally important to take into consideration the family perspective, the impact on family occupation along with the child's participation in different life situations.

\section{Rationale of the study:}

From the literature review and clinical experience following aspects are clear.

1. Sensory Processing Disorder is present in children with ADHD.

2. There is a wide use of Ayres Sensory Integration to treat children with ADHD.

3. There is no study on effect of ASI on participation of children with ADHD in life situations.

4. Most of the study does not evaluate the family perspective about the intervention.

5. There is no available tool to measure the level participation in children with ADHD.

In view of all these things, there is an urgent need to research the impact of ASI on children with ADHD. There is also a need to build a tool to assess the level of participation in life events. This would enable a potentially valuable contribution to patient care.

\section{Aims And Objectives:-}

The goal of the study is to investigate the impact of ASI ${ }^{\circledR}$ Interventions on the involvement of children. In addition, we would like to know the parent 's viewpoint on the effect of therapy in children diagnosed with ADHD. 


\section{Material and Methods:-}

\section{Trial design: Concurrent Mixed Methods Design}

The combination of qualitative and quantitative methods would allow to evaluate the effects of intervention from different perspectives. These are the assumptions of parents regarding behavioral improvement, the effect on child and family occupation, any limitation of activity and participation limits. In addition, mixed-method research would allow for the generation and verification of theory in a single experiment. (Figure 1)

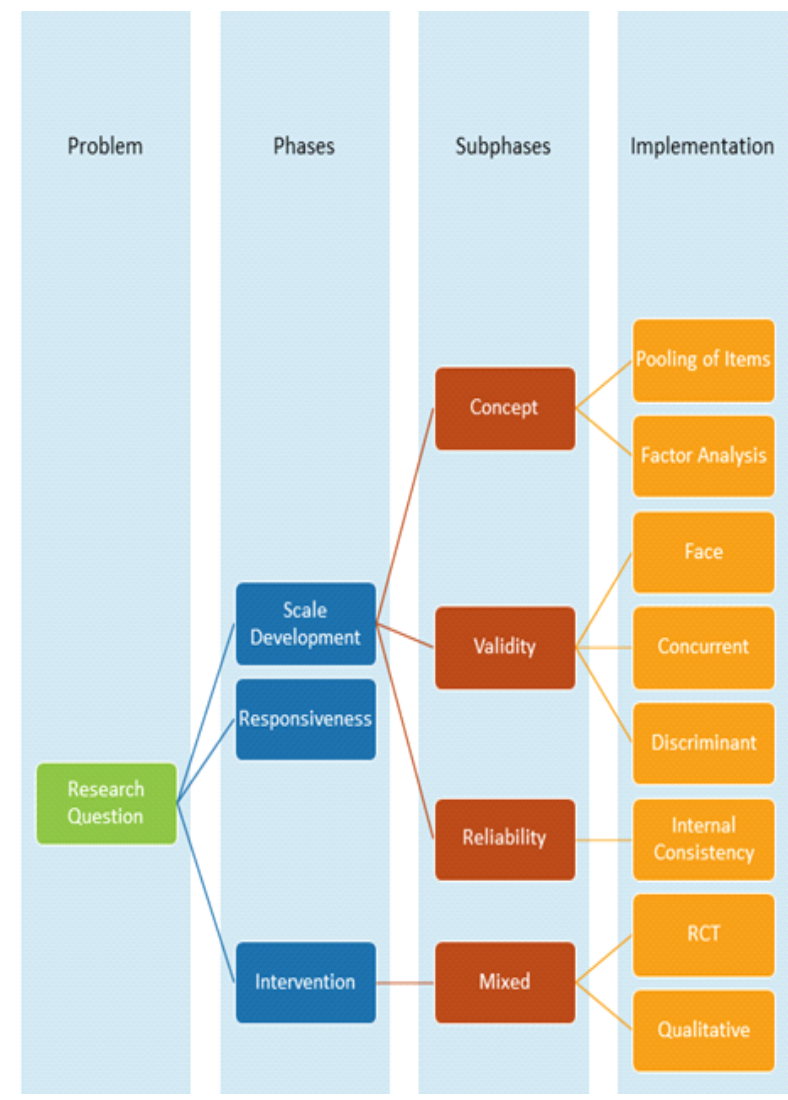

Figure 1:- Overview of Methodology.

All procedures performed were approved by Institutional Ethics Committee.

\section{Study 1 - Trial design:}

Psychometric design using cross section survey

\section{Participants \& recruitment:}

'Informed consent was obtained from all the individual participants participating in the study.' We conducted study at Occupational Therapy Department with parents of typically developing children and parents of children diagnosed with ADHD between the ages of 5 years and 12 years from Mumbai and Thane districts.

We recruited professionals from the different institutions in Mumbai to confirm the validity of the questionnaire. The participants were chosen by uniform sampling. We consulted 20 practitioners (Occupational Therapist, Pediatrician, Psychiatrist, and Psychologist) to check the face validity of the questionnaire.

In addition to the recently formed questionnaire (APP), we administered Sensory Processing Measure and ADHD rating scale IV: Home Type to test children before beginning Occupational Therapy.

\section{Study 2:}

Randomized controlled trial, double blind study

It was a two-group parallel trial. It was a double-blind study. 


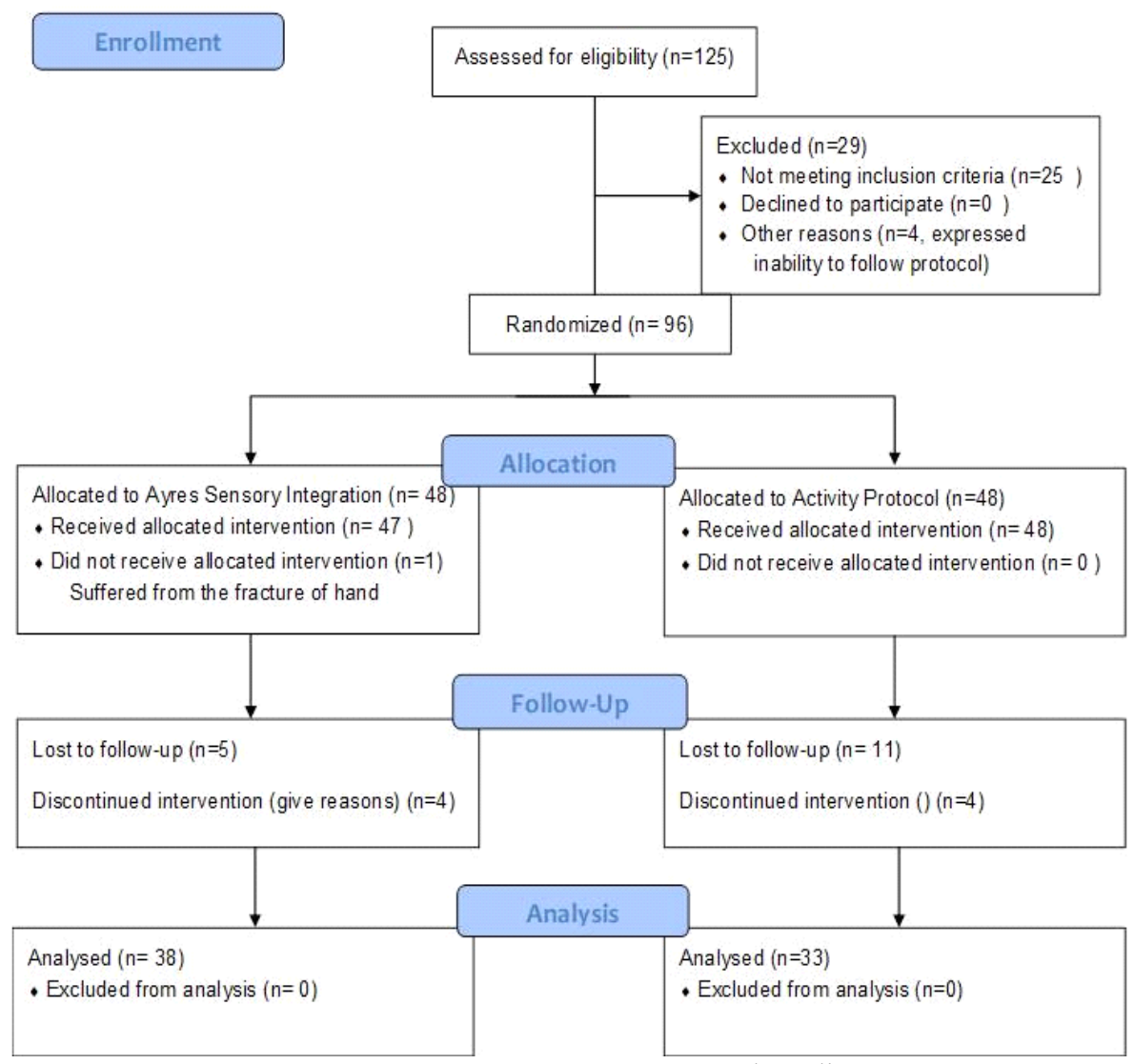

Figure 2:- Consort 2010 Flow diagram.

\section{Participants and their Recruitment}

Eligible candidates were children having average intelligence with a diagnosis of ADHD and between age of 5 years and 12 years. The diagnosis of ADHD was according to the DSM-IV TR guidelines and on ADHD Rating Scale Home Form score $\geq 80$ percentile. These children had sensory processing disorder.

\section{Study settings}

The study took place in department of Occupational Therapy of tertiary care Hospital.

\section{Interventions}

Children included in the study received occupational therapy with a frequency of 3 times per week for 6 weeks. During the study period the children received 18 sessions. In both the interventions procedure for evaluation, goal setting was same, to avoid confounding effect of the procedure.Experimental group received interventions based on Ayres Sensory Integration ${ }^{\circledR}$.

\section{Instruments Used}

\section{Screening instruments}

The ADHD Rating Scale-IV (Home Version) is a reliable and easy-to-administer instrument both for diagnosing ADHD in children and adolescents and for assessing treatment response.

Another screening measure was level sensory processing, Praxis and social participation as evaluated by parent rated Questionnaire viz. Sensory Processing Measure (SPM). SPM is a parent reported questionnaire that analyses functional behavior related to sensory processing. 


\section{Primary Outcome measure}

The primary outcome measure of the study was questionnaire viz. Participation Profile, which measures the level of participation in life situations (Dependent Variable). For each item the gradation of difficulty for the child was No (0), mild (1), moderate (2), complete (3) and severe (4); (Ordinal scale). This level of difficulty was rated by parents/ caretakers.

\section{Secondary Outcome measures}

Also, functional improvement was evaluated by Goal Attainment Scale. For setting up goals of the treatment, Goal Attainment Scale (GAS) was used.

\section{Sample size}

A total of 96 patients entered this two-treatment parallel-design study. The probability is 90 percent that the study will detect a treatment difference at a two-sided 0.05 significance level, if the true difference between treatments is 0.670 times the standard deviation.

\section{Responsiveness}

After completing the APP questionnaire, the intervention commenced. In the present study, the Ayres Sensory Integration ${ }^{\circledR}$ Therapy method offered by the Occupational Therapist at Sensory Integration Therapy clinic. The therapy was given to each participant for six weeks. After the intervention six weeks period, they were required to fill in the ADHD Participation Profile questionnaire again. The responsiveness of the ADHD Participation Profile questionnaire was determined using the effect size and the standardized response mean (SRM).

\section{Study 3:}

Qualitative study, phenomenology - Family perspective was evaluated by qualitative data (interview method).

\section{Results:-}

Detailed characteristics of participants are in Table 1.

Table 1:- Characteristics of the Study Participants for APP development.

\begin{tabular}{|c|c|}
\hline Item & Value \\
\hline Typically Developing Children & 23 \\
\hline Total Number of children & 19 \\
\hline Male & 4 \\
\hline Female & 8.19 years $( \pm 1.85)$ \\
\hline Average Age (Mean \pm Standard Deviation) & 93 \\
\hline Children with Diagnosis of ADHD & 72 \\
\hline Total Number of Children & 21 \\
\hline Male & 7.52 years $( \pm 2.3)$. \\
\hline
\end{tabular}

Table 2 shows the age and gender distribution along with sensory processing issues of participants in the study.

Table 2:- Sensory Processing issues as per Sensory Processing Measure.

\begin{tabular}{|l|l|l|l|l|l|l|l|l|}
\hline $\begin{array}{l}\text { SPM } \\
\text { DOMAINS }\end{array}$ & SOC & VIS & HEAR & TOUCH & BODY & BAL & PLA & TOT \\
\hline MEAN & 31.52 & 15.90 & 9.62 & 17.84 & 17.09 & 17.48 & 18.71 & 134.20 \\
\hline SD & 5.99 & 4.21 & 2.07 & 5.06 & 4.99 & 3.42 & 6.38 & 16.90 \\
\hline $\begin{array}{l}\text { No. of } \\
\text { children } \\
\text { with }\end{array}$ & 68 & 7 & 0 & 8 & 10 & 6 & 19 & 77 \\
$\begin{array}{l}\text { Definite } \\
\text { Dysfunction }\end{array}$ & 70.83 & 7.29 & 0 & 8.33 & 10.41 & 6.25 & 19.79 & 80.20 \\
\hline $\begin{array}{l}\text { \% of } \\
\text { children } \\
\text { with DD }\end{array}$ & & & & & & & \\
\hline
\end{tabular}




\begin{tabular}{|l|l|l|l|l|l|l|l|l|}
\hline $\begin{array}{l}\text { No. of } \\
\text { children } \\
\text { with Some } \\
\text { Problems }\end{array}$ & 92 & 44 & 17 & 52 & 52 & 53 & 55 & 96 \\
\hline $\begin{array}{l}\text { \% of } \\
\text { children } \\
\text { with Some } \\
\text { Problems }\end{array}$ & 95.83 & 45.83 & 17.70 & 54.16 & 54.16 & 55.20 & 57.29 & 100 \\
\hline
\end{tabular}

Table 3:- Responsiveness of APP from baseline.

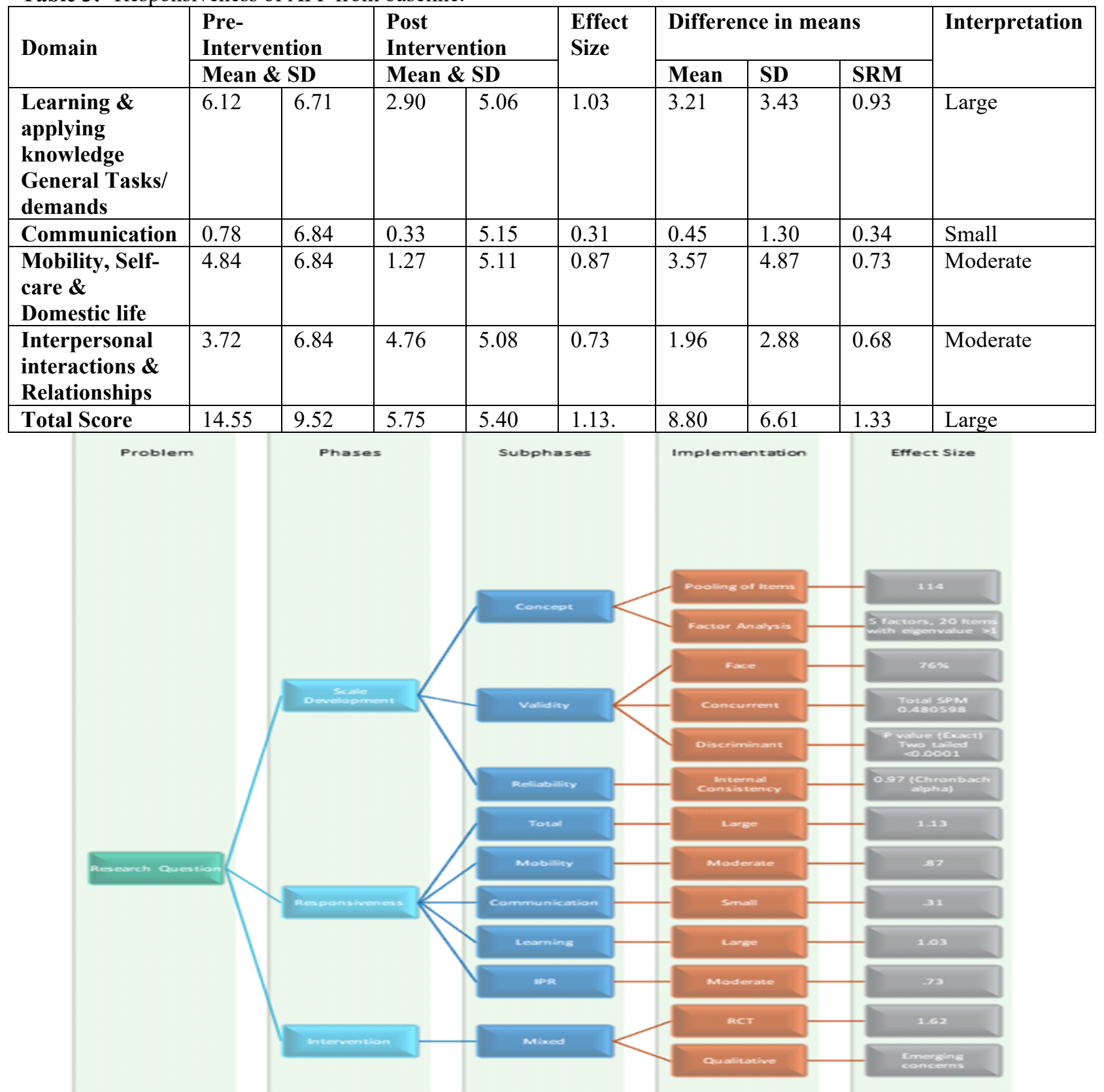

Qualitative

Figure 3: - Overview of effect sizes.

Analysis and findings

Data analysis: 
The coding was done by four coders including principal investigators. Total numbers of participants were 40 (33 boys +7 girls). We derived 175 codes. We prepared theme after coding (Figure 7).

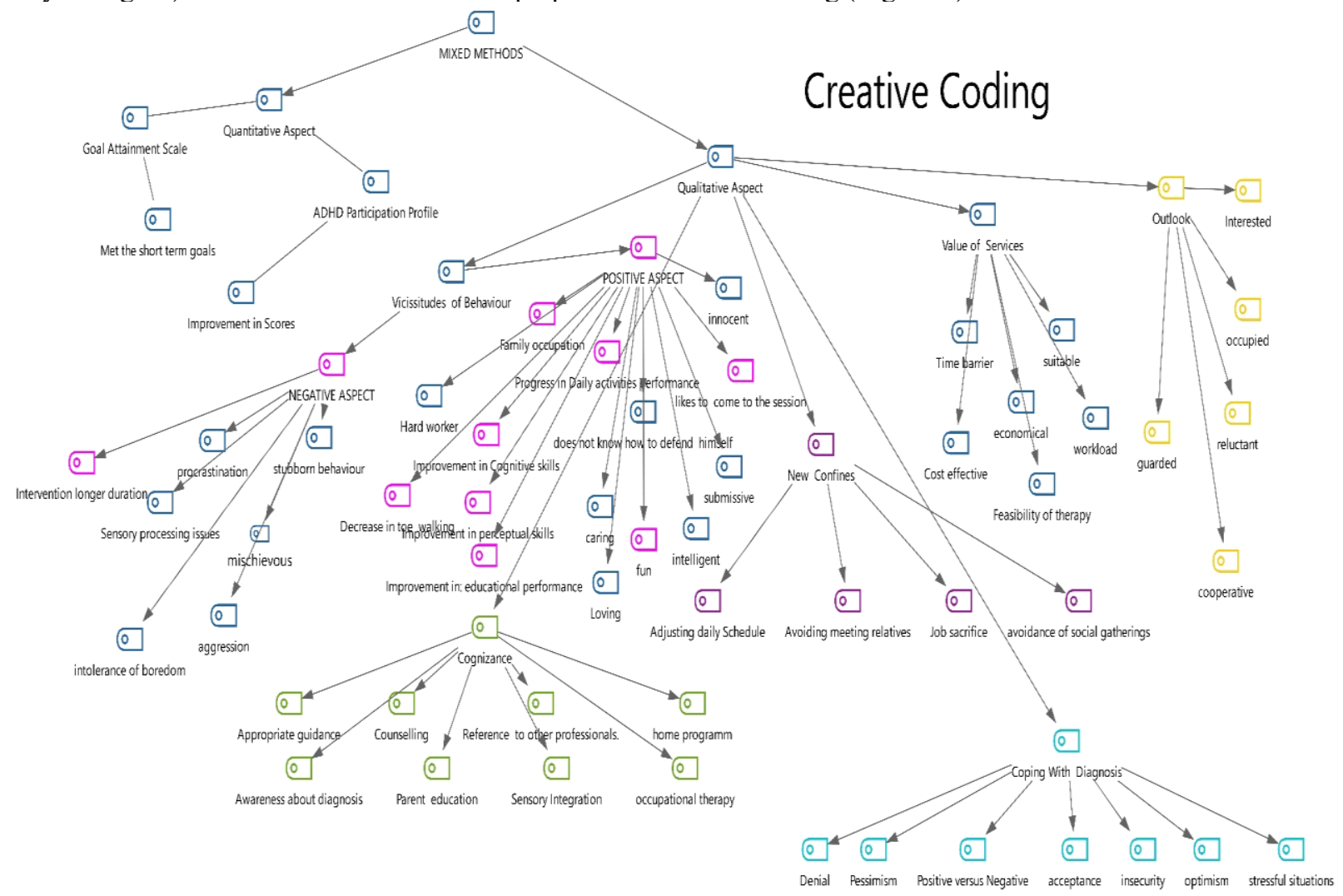

Figure 4:- Map of Codes in context with the study.

\section{Discussion:-}

The goal of the Initial study was to obtain a profile of children diagnosed with ADHD. This profile was intended to measure the parent 's view of the child's level of difficulty in engaging in everyday chores. We have gathered the evidence for the final profile via a newly developed questionnaire. The present study determined the validity and reliability of the newly drafted questionnaire viz. ADHD Profile of Participation. This questionnaire assesses the extent of participation in children diagnosed with ADHD from the point of view of the parent.

We used the ADHD Participation Profile (APP) Factor Analysis to show the primacy of parents' interpretation. The study of the factor resulted in the discovery of 13 factors. Out of 13, we picked five variables, which included the majority of items. In conclusion, we selected a total of 20 items.

We computed Cronbach's alpha for the final version of the ADHD Participation Profile after validation of the construct and was 0.97 . It is signifying the strong correlation between the items and the questionnaire is reliably accurate. As a result, clinicians should use the ADHD Participation Profile in regular client education and management. For example, clinicians may use it comfortably in their regular clinical practice to incorporate the level of participation in the care of their clients.

There was a considerable difference between the scores obtained from parents of children diagnosed with ADHD and parents of typically developing children $(\mathrm{p}<0.001)$. This suggests the ADHD Participation Profile has been able to discriminate parents' view of typically developing children and children with a diagnosis of ADHD.

The results of the Responsiveness Study indicate that the ADHD Participation Profile questionnaire is responsive and may track the results of the treatment. In conclusion, the responsiveness of the ADHD Participation Profile questionnaire was shown to be high. Herein, the ADHD Participation Profile can be used accurately to monitor improvement in the care of ADHD patients for research and clinical purposes. 
As Dr. David Ben points out, Sensory Processing Problems are also part of the diagnosis and may be considered a common co-morbidity with ADHD. The best way is therefore to handle all conditions at the same time as accepted treatment protocols.

The findings of the RCT show that ASI ${ }^{\circledR}$ is successful in alleviating the concerns of the family and children with ADHD. Children in the ASI ${ }^{\circledR}$ group also made significant changes to the Activity Protocol Group on the Goal Attainment Scale and the ADHD Participation Profile.

The combination of therapies will definitely improve efficiency compared to individual therapies using just one approach. A large multidimensional intervention approach consisting of sensory integration and other non-sensory psychosocial interventions decided on the need for individual priorities could be a better choice.

Goal Attainment Scaling and ADHD Participation Profile were complementary in their ability to assess individual improvements over time in children with ADHD. Using ADHD Participation Profile alone may have caused many of the individual recovery targets that were ultimately reached to be missed in the outcome assessment.

We used only the hypothesis-driven measure of the result. Participation was the purpose of the intervention. For this reason, we used ADHD Participation Profile which is a true, reliable and sensitive instrument. ADHD Participation Profile is primarily intended as an outcome measure for monitoring progress.

Qualitative research has been performed with the goal of studying the viewpoint of Caretakers on the impact of occupational therapy with Ayers Sensory integration on children diagnosed with ADHD.

In the qualitative process, the explanations provided by the caregivers demonstrate the degree to which the child is engrossed and how it affects the functioning of the individual caregiver and the family. Harpin claimed that parents are under stress when handling a child in difficult circumstances and how ADHD adversely affects the equation within the family.

In the background of the effects of Occupational Therapy, the outcomes were mostly positive and how Occupational Therapy training based on Sensory Integration Therapy helped to resolve children's and family occupation issues.

But after reflecting on the perennial worries of the parents, we realized that the impact was reassuring. While shortterm effects have been confirmed, they are not permanent measures. Hinshaw also concluded that intervention for ADHD must be treated as a lifetime undertaking (Hinshaw, 2015).

There have been new limits of family occupation in the form of sensory dietary guidelines. However, the need for the hour recommends adjusting daily conditions to meet the needs of children with various patterns of sensory processing. As set out in, family occupations are necessarily manifold, changing and negotiated. Blending therapeutic services into everyday life involves attention to the dynamics of family interaction. Families, too, seldom have exclusive modes of action directed at them. It might not be enough to provide a dedicated time of day or week to "suit" health measures (Miao, 2017). Families are culturally unique communities that need customized support for incorporating these strategies.

Practitioners may also need to consider how these strategies will overlap, connect and spread through the general activity environment at home. The last theme has been connected to awareness. This offered an evaluation of the diagnosis; intervention and other causes. Indeed, an endowment applicable to all aspects was required. Considering the complex nature of the disorder, there have been a variety of treatments. Parents were expecting more enlightenment in this area. At the end of the qualitative enquiry, Rana (Ahmed, 2014) also found that "there are gaps in parents' knowledge of ADHD and its treatment and an expressed need for personalized and accurate information."

We conclude that Occupational Therapy has brought a major improvement in the lives of children and parents. While certain targets are reached after the intervention, the list of apprehensions is endless. Many parents were less worried after occupational therapy, though few were still uncertain about the future of their children. 
Quantitative methods have its own limitations. It cannot always represent the actual happening. Families had real challenges and they were many-sided. We would have ignored this ironic data if we had to offer a judgement on the impact of the intervention by means of a quantitative measure. Mixing the approaches was an appropriate answer.

\section{Summary \& Conclusion:-}

ADHD is not simply a matter of sensory processing. It is a complex disorder that requires issues other than sensory processing and may be sensitive to other types of intervention. If present, the issues of sensory processing need to be discussed explicitly, as they can have an effect on overall performance. The mixed approach style for managing ADHD was also found to be balancing for limiting the lacunae of either qualitative or quantitative approaches.

\section{Mixed Method Approach to ADHD Management}

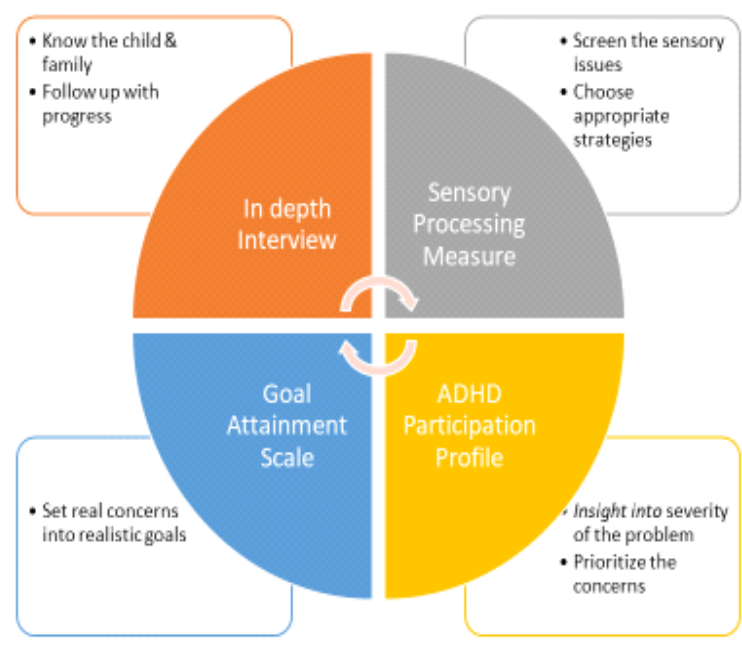

\section{References:-}

1. Abele-Webster L, M.-E. J. (2012). Sensory processing and ADHD in children with fetal alcohol spectrum disorder. The Canadian Journal of Occupational Therapy, 79(1), 60.

2. Ayres AJ, M. Z. (1987). Developmental Dyspraxia: Is It A Unitary Function? OTJR, 7(2), 93-110.

3. B. French, K. S. (2019). Barriers and facilitators to understanding of ADHD in primary care: a mixed-method systematic review. European Child \& Adolescent Psychiatry, 1037-1064.

4. Chu S, R. F. (2007). Occupational therapy for children with attention deficit hyperactivity disorder (ADHD), part 2: a multicentre evaluation of an assessment and treatment package. British Journal of Occupational Therapy, 439-48.

5. Creswell, J. W. (2009). Research Design. In J. W. Creswell, Research Design: Qualitative, Quantitative, and Mixed Methods Approaches (Third Edition ed.). Lincoln, University of Nebraska: SAGE Publications, Inc.

6. Davies PL, T. R. (2010). Evidence Review to Investigate the Support for Subtypes of Children With Difficulty Processing and Integrating Sensory Information. The American Journal of Occupational Therapy, 64(3), 391-402. 
7. Dr David Benn, P. (2017, June 1). ADHD and Sensory Integration Disorders. MENTAL HEALTH MATTERS, p. 3. Retrieved March 17, 2020, from http://www.sadag.org/images/pdf/ADHD-and-sensoryintegration-disorders.pdf

8. Dunn W, B. D. (2002). Patterns of sensory processing in children with attention deficit hyperactivity disorder. OTJR, 22(1), 4-15.

9. DuPaul, G. J. (1991). Parent and teacher ratings of ADHD symptoms: psychometric properties in a communitybased sample. $J$ Clin Child Adolesc Psychol, 20(3), 245-253. doi:https://doi.org/10.1207/s15374424jccp2003_3

10. Farin, E. U. (2013). Participation and social functioning in patients with fibromyalgia: Development and testing of a new questionnaire. Health and Quality of Life Outcomes, 11, 135.

11. Fedewa, A. L. (2011). Stability balls and students with attention and hyperactivity concerns: Implications for on-task and in-seat behavior. The American Journal of Occupational Therapy, 65(4), 393-9.

12. Frick, P. J. (2010). Assessment of Attention Deficit Hyperactivity and Disruptive Disorders (3 ed.). (P. J. Frick, Ed.) London: Springer.

13. Ghanizadeh, A. (2011). Sensory Processing Problems in Children. with ADHD, a Systematic Review. Psychiatry Investig, 89-94.

14. Ghuman JK, A. L. (2008). Psychopharmacological and Other Treatments in Preschool Children with Attention-Deficit/Hyperactivity Disorder: Current Evidence and Practice. $J$ Child Adolesc Psychopharmacol, 18(5), 413-47.

15. Gol D, J. T. (2005). Effect of a social skills training group on everyday activities of children with attentiondeficit hyperactivity disorder. Dev Med Child Neurol, 47(8), 539-45.

16. Gupta, S. K. (2011). Intention-to-treat concept: A review. Perspectives in Clinical Research, 109-112.

17. Hemant Nandgaonkar, Z. F. (2016). Endorsement: Ayres Sensory Integration ${ }^{\circledR}$ for Attention Deficit and Hyperactivity Disorder. Indian Journal of Applied Research, 507.

18. Hemant, N. (2015). Gestalt of non-pharmacological management of ADHD. International Journal of Current Research and Academic Review, 156-181.

19. Hongwanishkul, D. (2005). Assessment of Hot and Cool Executive Function in Young Children: AgeRelated Changes and Individual difference. DEVELOPMENTAL NEUROPSYCHOLOGY, 617-644.

20. http://hedwig.mgh.harvard.edu/sample_si ze/js/js_associative_quant.html. (n.d.).

21. Janice A. Husteda, R. J. (2000). Methods for assessing responsiveness: a critical review. Journal of Clinical Epidemiology, 53, 459-468.

22. Johnston, C. \&. (2015, March). Interventions for Attention-Deficit Hyperactivity Disorder: A Year in Review. Current Developmental Disorders Reports, 2(1), 38.

23. Kazis LE, A. J. (1989). Effect sizes for interpreting changes in health status. Med Care, 27(Suppl 3), S17889.

24. L. Diane Parham, S. S.-B.-G. (2011). Development of a Fidelity Measure for Research on the Effectiveness of the Ayres Sensory Integration Intervention. American Journal of Occupational Therapy, 65, 133.

25. Lenhard, W. \&. (2016, 1 1). Calculation of Effect Sizes. :DOI: 10.13140/RG.2.1.3478.4245. Retrieved 9 29, 2018, from Psychometrica: https://www.psychometrica.de/effect_size.html.

26. M. Huizinga, C. D. (2006). Age-related change in executive function: Developmental trends and a latent variable analysis. Neuropsychologia, 2017-2036.

27. Man Hung, C. L. (2017). The responsiveness of the PROMIS instruments and the qDASH in an upper extremity population. Journal of Patient-Reported Outcomes, 1-13.

28. Mohd Normani Zakaria, P. W. (2018). Test-retest reliability and responsiveness of a Malay tinnitus questionnaire. Med J Malaysia, 73(1), 7.

29. Nandgaonkar Hemant P., F. Z. (2017). Attention Deficit \& Hyperactivity Disorder (ADHD) Participation Profile (APP): Primacies in Parent's Perception. INDIAN JOURNAL OF APPLIED RESEARCH, 170.

30. Nandgaonkar Hemant, F. Z. (2018, August). Responsiveness Of APP: Attention Deficit \& Hyperactivity Disorder (ADHD) Participation Profile. Indian Journal of Applied Research, 8(8), 354-355.

31. Nandgaonkar HP, F. Z. (2017). Attention Deficit \& Hyperactivity Disorder (ADHD) Participation Profile (APP): Primacies in Parent's Perception. Indian Journal of Applied Research.

32. OBrien JC, W. H. (2008). Mechanisms That Underlie Coordination in Children With Developmental Coordination Disorder. J Mot Behav, 40(1), 43-61.

33. Pappas, D. (2006). ADHD Rating Scale--IV (for Children and Adolescents): Checklists, Norms, and Clinical Interpretation. Journal of Psychoeducational Assessment , 172. 
34. Rainey, L. v. (2014). Measurement properties of questionnaires assessing participation in children and adolescents with a disability: A systematic review. Quality of Life Research, 23(10), 2793-808.

35. Schilling, O. L. (2003). Classroom seating for children with attention deficit hyperactivity disorder: Therapy balls versus chairs. , 57, . American Journal of Occupational Therapy, 534-541.

36. Schilling., D. L. (2004). Examining alternative seating devices for children with attention deficit hyperactivity disorder: Effects on classroom behavior. . United States -- Washington: United States -Washington: University of Washington.

37. Schulz KF, A. D. (2010). CONSORT 2010 Statement: updated guidelines for reporting parallel group randomized trials. . Trials 2010, CONSORT Group. .

38. Segal, R. (1998). The construction of family occupations: A study of families with children who have Attention Deficit/Hyperactivity Disorder. Cananda: CAOT PUBLICATIONS ACE.

39. Shanley DM, M. L.-C. (2001). Sensory modulation dysfunction in children with attention-deficithyperactivity disorder. Dev Med Child Neurol, 43(6), 399-406.

40. Terwee CB, B. S. (2007). Quality criteria were proposed for measurement properties of health status questionnaires. J Clin Epidemiol, 60(1), 34-42.

41. VA., H. (2005). The effect of ADHD on the life of an individual, their family, and community from preschool to adult life. Arch Dis Child, 90.

42. Vandana J Rathod, V. S. (2015). Effect of sensory integration therapy and cognitive behavioral therapy on attention deficit. International Journal of Physiotherapy and Research, 947.

43. Verhoef, J. T.-S. (2008). Validity and responsiveness of the rehabilitation activities profile (RAP) in patients with rheumatoid arthritis. Clinical Rehabilitation, 22(9), 788-800.

44. Watemberg N, W. N.-S. (2007). Developmental coordination disorder in children with attention-deficithyperactivity disorder and physical therapy intervention. Dev Med Child Neurol, 920.

45. Wilkes-Gillan S, B. A. (2016). A Randomised Controlled Trial of a Play-Based Intervention to Improve the Social Play Skills of Children with ADHD. PLoS ONE, 1-22. 